\title{
UCRL-TR-231593
}

LAW RENCE LIVERMORE N A TIO N A L LABORATORY

\section{Thermal Radiation from Nuclear Detonations in Urban Environments}

R. E. Marrs, W. C. Moss, B. Whitlock

June 7, 2007 
This document was prepared as an account of work sponsored by an agency of the United States Government. Neither the United States Government nor the University of California nor any of their employees, makes any warranty, express or implied, or assumes any legal liability or responsibility for the accuracy, completeness, or usefulness of any information, apparatus, product, or process disclosed, or represents that its use would not infringe privately owned rights. Reference herein to any specific commercial product, process, or service by trade name, trademark, manufacturer, or otherwise, does not necessarily constitute or imply its endorsement, recommendation, or favoring by the United States Government or the University of California. The views and opinions of authors expressed herein do not necessarily state or reflect those of the United States Government or the University of California, and shall not be used for advertising or product endorsement purposes.

This work was performed under the auspices of the U.S. Department of Energy by University of California, Lawrence Livermore National Laboratory under Contract W-7405-Eng-48. 


\title{
Thermal Radiation from Nuclear Detonations in Urban Environments
}

\author{
R. E. Marrs, W. C. Moss, and B. Whitlock \\ Lawrence Livermore National Laboratory
}

\section{Introduction}

There are three principal causes of "prompt" casualties from a nuclear detonation: nuclear (gamma-ray and neutron) radiation, thermal radiation, and blast. Common estimates of the range of these prompt effects indicate that thermal radiation has the largest lethal range [1]. Non-lethal skin burns, flash blindness, and retinal burns occur out to much greater range. Estimates of casualties from thermal radiation assume air bursts over flat terrain. In urban environments with multiple buildings and terrain features, the extent of thermal radiation may be significantly reduced by shadowing.

We have developed a capability for calculating the distribution of thermal energy deposition in urban environments using detailed 3D computer models of actual cities. The size, height, and radiated power from the fireball as a function of time are combined with ray tracing to calculate the energy deposition on all surfaces. For surface bursts less than $100 \mathrm{kt}$ in locations with large buildings or terrain features, the calculations confirm the expected reduction in thermal damage.

\section{Fireball Behavior}

\section{Air Burst}

The optical (and thermal) radiation from a free-air nuclear burst at low altitude displays a characteristic double pulse. The first pulse is much shorter than the second and contains roughly $1 \%$ of the total thermal energy, so it is not important for the calculation of thermal radiation. The second pulse begins as the shock front cools and becomes transparent, revealing the hot fireball behind it. The second pulse contains nearly all of the thermal energy. In the present work, we use approximate expressions for the time dependence of the radiated power, radius, and height of the fireball during the second pulse to calculate the distribution of thermal radiation.

A single normalized thermal power curve, shown in Fig. 1, applies for all yields when plotted as a function of normalized time $\tau=t / t_{\max }$ where $t_{\max }$ is the time of peak power [1]. We have fitted the empirical power curve of Ref. 1 with the function

$$
P(\tau)=\frac{2.85(\tau+0.5)^{-2}}{1+e^{-(\tau-0.8) / 0.15}} .
$$

The time of peak power is $t_{\max }=0.04 W^{0.44}$ seconds, where $W$ is the yield in kt, and the peak power is $P_{\max }=3.2 \times 10^{12} W^{0.56} \mathrm{cal} / \mathrm{s}$ [1]. With these expressions for $t_{\max }$ and $P_{\max }$, the integral of $P(\tau)$ from $t=0$ to $\infty$ is $0.29 \mathrm{~W}$, corresponding to $29 \%$ of the yield in radiated thermal energy. The accuracy of the power curve is estimated to be $\pm 25 \%$ [1]. 


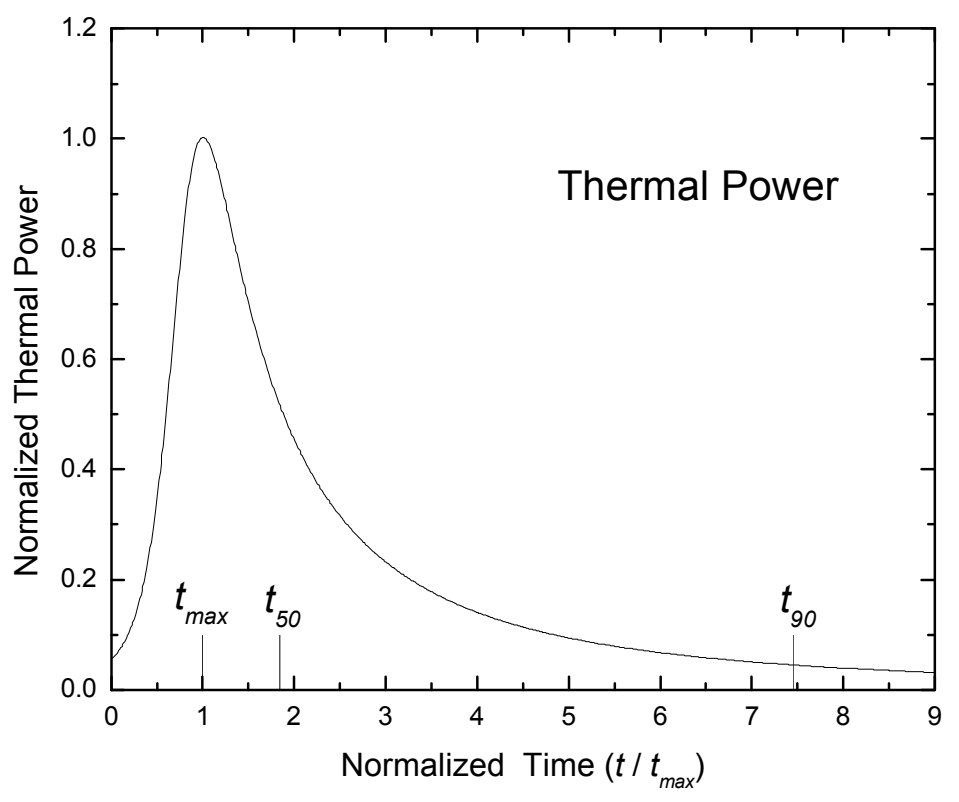

Fig. 1. Power curve for thermal radiation from a nuclear air burst as given by Eq. 1 . Times at which $50 \%$ and $90 \%$ percent of the total power has been radiated are indicated.

It is difficult to find a good formula for the size of the thermal radiation source. The visual fireball radius is a good estimate of where the escaping thermal radiation originates. The visual fireball radius increases during the rising part of the power curve, and then collapses rapidly after peak power as the hot air cools to transparency, revealing the smaller, still-radiating hot debris cloud. At this point, roughly half the thermal radiation has been emitted. Symbalisty, Zinn, and Whitaker have calculated the visual radius vs. time with the RADFLO code and find that it has a broad maximum near the time of peak thermal power [2]. The maximum radius is given approximately by $R_{\max }=$ $50 \mathrm{~W}^{1 / 3}$ meters. Another common estimate of fireball size is the radius at the time of shock breakaway, which marks the beginning of the second optical pulse. This radius is $R_{\text {breakaway }}=34 W^{0.4}$ meters [1]. Instead of trying to construct an (inaccurate) time dependent model of the size of the thermal source, we use a spherical surface of constant radius given by the value at peak power, $R=50 \mathrm{~W}^{1 / 3}$.

Even though most of the thermal energy from a nuclear burst is radiated quickly, the buoyant rise of the hot fireball is important for large yields or irradiation of areas that would otherwise be in the shadows of buildings or terrain. We have included the fireball rise in our model with the formula

$$
\frac{d H}{d t}=50 W^{0.19} t^{-0.18} \mathrm{~m} / \mathrm{s}
$$

where $H$ is the height of the fireball [3]. The fireball height is plotted in Fig. 2, and values for fireball thermal properties for selected yields are given in Table I. 


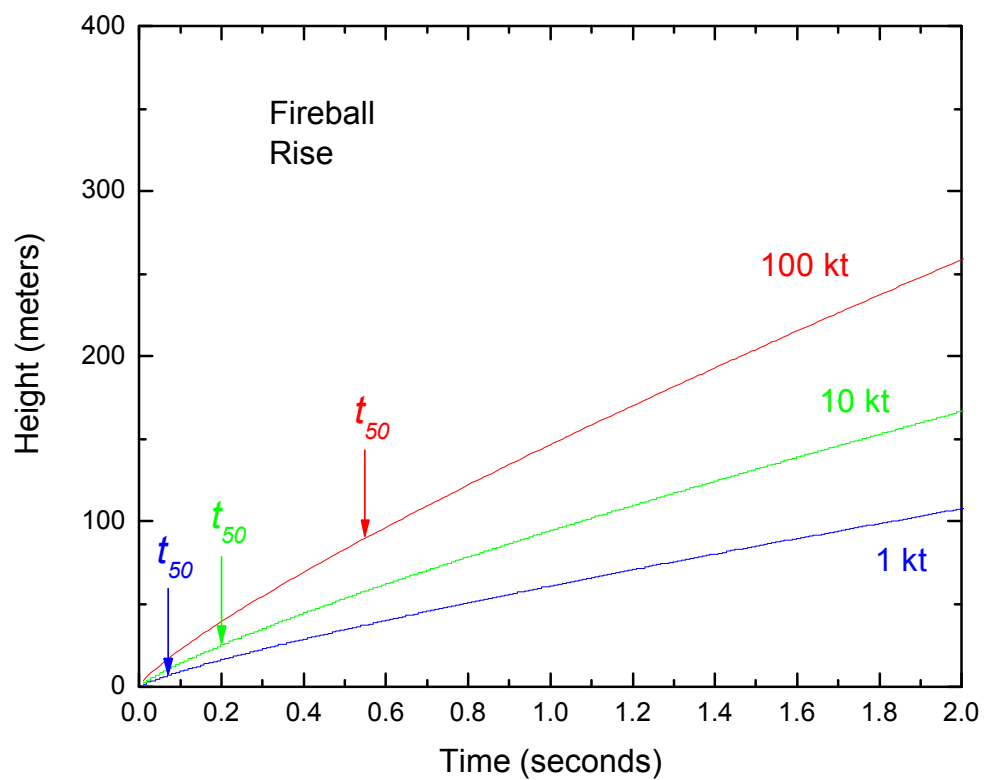

Fig. 2. Height of fireball center above burst point for different yields as indicated. Arrows indicate the time at which $50 \%$ of the thermal radiation has been emitted.

Table I. Fireball parameters for yields of 1,10 , and $100 \mathrm{kt} . t_{\max }$ is the time of peak power. $t_{50}$ and $t_{90}$ are the times at which $50 \%$ and $90 \%$ of the thermal energy has been radiated.

\begin{tabular}{cccccccc}
$\begin{array}{c}\text { Yield } \\
(\mathrm{kt})\end{array}$ & $\begin{array}{c}t_{\max } \\
(\mathrm{s})\end{array}$ & $\begin{array}{c}t_{50} \\
(\mathrm{~s})\end{array}$ & $\begin{array}{c}t_{90} \\
(\mathrm{~s})\end{array}$ & $\begin{array}{c}\text { Radius } \\
(\mathrm{m})\end{array}$ & $\begin{array}{c}\text { Height at } \\
t_{50}(\mathrm{~m})\end{array}$ & $\begin{array}{c}\text { Height at } \\
t_{90}(\mathrm{~m})\end{array}$ & $\begin{array}{c}\text { Peak Power } \\
\left(10^{12} \mathrm{cal} / \mathrm{s}\right)\end{array}$ \\
\hline 1 & 0.04 & 0.07 & 0.30 & 50 & 7 & 23 & 3.2 \\
10 & 0.11 & 0.20 & 0.82 & 108 & 25 & 80 & 12 \\
100 & 0.30 & 0.55 & 2.2 & 232 & 90 & 284 & 42
\end{tabular}

\section{Surface Burst}

The properties of a fireball and its thermal radiation may be significantly altered if the burst occurs on the surface, within a massive structure (e.g., truck or garage), or underground. A common procedure for treating surface bursts uses the expressions for the properties of an air burst with the yield twice as large as the actual yield. This accounts for the fact that the thermal and blast energy must now go into $2 \pi$ steradians instead of into $4 \pi$. 
Our code accepts an arbitrary height of burst, and some approximations are required to handle bursts near the surface. We calculate fireball parameters from the actual yield (not $2 \times$ yield) and use a spherical fireball shape, even for near-surface bursts. However for heights of burst between zero and $0.4 R$ above the surface, where $R$ is the fireball source radius as given above, the center of the spherical fireball is placed at $0.4 R$ above the surface.

We model subsurface bursts by raising the position of the fireball by $0.4 R$, moving it closer to the surface to partially account for the behavior of these bursts, for which the fireball erupts through the surface. (See discussion of the source model below.) This means that for a depth of burst greater than $1.4 R$, the spherical fireball source in our model will be completely below the surface and there will be no thermal radiation. For higher burst points, only the fraction of the fireball that is above the surface contributes to the thermal radiation.

The actual thermal radiation from a surface burst, and especially from a partially buried burst, is difficult to predict because of the large amount of solid material mixed into the fireball and lofted near the burst point. This produces a cooler fireball and attenuates the thermal radiation so that the radiated thermal energy may be significantly less than that from an air burst. In Ref. 4, the thermal energy fraction for a surface burst is estimated to be $50 \%$ of that for an air burst, and in Ref 1 it is estimated to be 50 to $75 \%$. This reduction in thermal radiation for a surface burst compensates the $2 \mathrm{~W}$ increase mentioned above and suggests that our choice of thermal radiation with yield $W$ is reasonable. An accurate calculation of thermal radiation from a surface or buried burst is beyond the scope of the present work - our focus is the shadowing effect of urban terrain.

\section{Digital City Models}

The ArcGIS software from ESRI corporation is a geographic information system that has become a widely used standard [5]. Digital shape files for many cities as well as digital terrain models are available in ArcGIS format. Our computer code accepts urban models in ArcGIS format. The models provide external building shapes, but they do not include structural details such as windows. Here we present results for two different digital city models: Oklahoma City with flat terrain, and San Francisco with hilly terrain. A digital model of San Francisco, as used in the thermal radiation calculations, is shown in Fig. 3. 


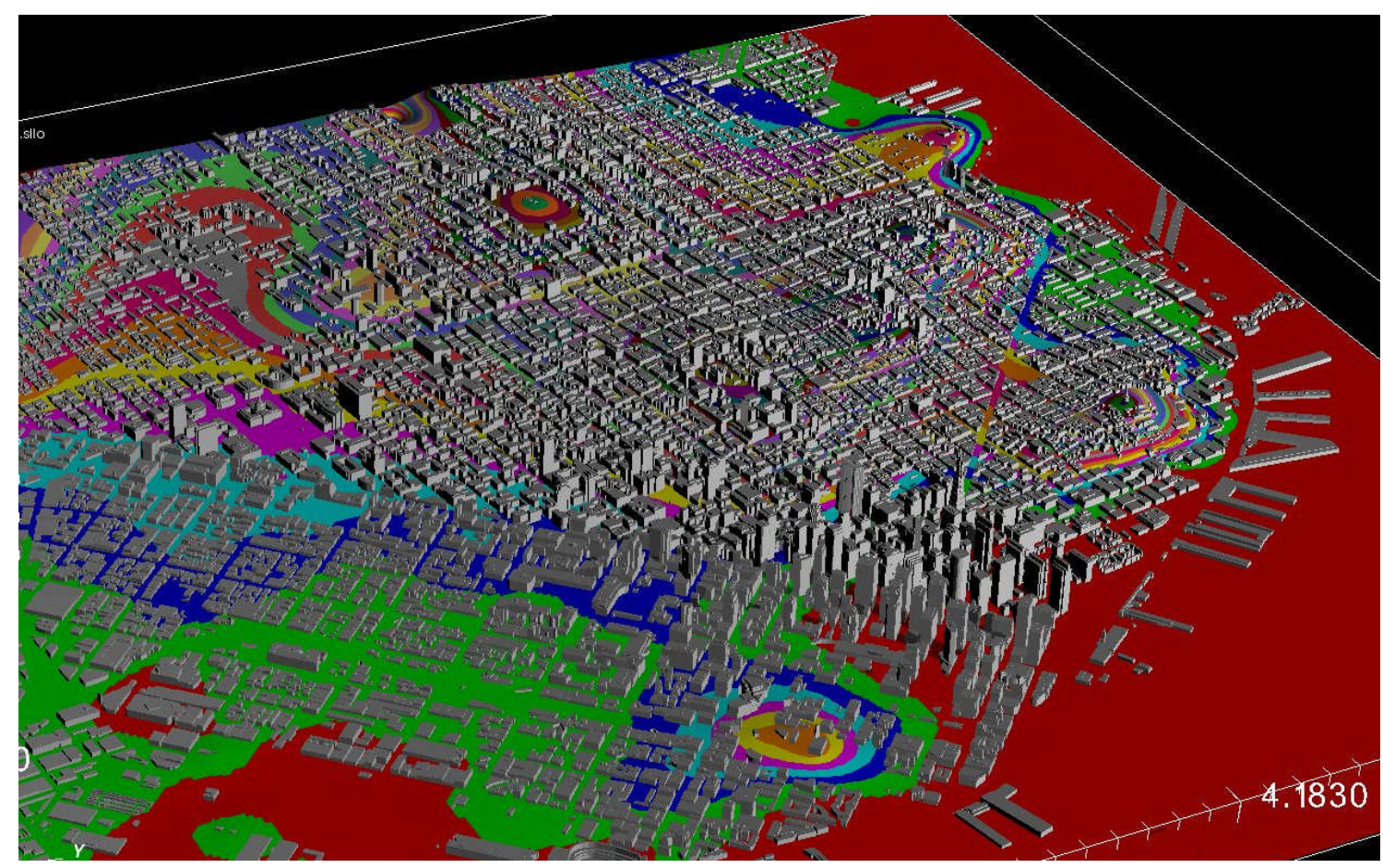

Fig. 3. Digital model of San Francisco buildings and terrain. Terrain elevation contours are color coded.

\section{Ray Tracing}

The thermal ray tracing is implemented in two separate codes that run sequentially. The first code, citify, reads the ArcGIS shape files and constructs an appropriate mesh. The second code, cityray, contains the fireball model and does the ray tracing. During the ray tracing, each element of the mesh receives an incremental heat deposition proportional to its solid angle with respect to the source. The incremental heat depositions are tallied for multiple time steps on the thermal power curve. There is also an integration over the finite size of the source as described below.

\section{Mesh Generation}

The ArcGIS digital models of buildings and terrain (if needed) are converted to a triangular mesh that covers all surfaces. The roughly one-meter size of the triangles (depending on the overall size of the model) determines the spatial resolution of the calculation. Each triangular cell accumulates thermal energy uniformly within the cell.

\section{Source Model}

Because of the large size of the spatial mesh and the integration over multiple time steps, an additional high-resolution integration over the finite source size would 
require long running times and is not justified by the overall accuracy of the model. Hence we chose a 13-step integration over the spherical fireball source.

For each cell of the surface mesh, the source was approximated as a disc with the same center and radius as the fireball and oriented perpendicular to the line of sight from the center of the cell to the center of the fireball. For distances large compared to the fireball radius, this is an excellent approximation to the actual spherical fireball surface. (A good analogy is the appearance of the sun from earth.) The integration over the radiating surface of the disc was done by dividing the disc into 13 segments of equal area as shown in Fig. 4. Each segment was approximated as a point source located at the center of the segment. Note that, except for the center segment, the disc of 13 source points is different (rotated) for each irradiated zone because the disc is oriented to face each irradiated zone separately.

The choice of a minimum height of burst of $0.4 R$ for a near-surface above ground burst (see the surface burst discussion above) puts 11 of the 13 distributed source points above ground so that the radiated thermal energy is $11 / 13$ of what it would be for a dustfree, clear-air burst. For a buried burst, fewer of the source points may be above ground. If none of them is above ground, the model gives zero thermal radiation.

Thermal irradiation of the mesh cells is tallied by ray tracing from the center of the mesh cell to each of the 13 source points and summing their contributions. The size of the contribution is proportional to the solid angle that the cell subtends with respect to a source point. A straight line (ray) from the center of a cell to a source point determines whether that cell receives zero or the full radiation contribution according to whether the line intersects a building or terrain. Time integration over the radiated power curve is done with a selectable number of time steps (typically 30 ) from $t=0$ to roughly $t_{90}$.

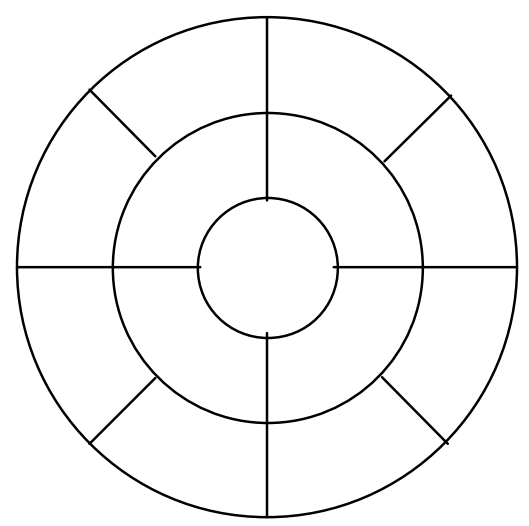

Fig. 4. Segmented source disc as seen from an irradiation zone. The outer radius is the fireball radius $R=50 W^{1 / 3} \mathrm{~m}$. 


\section{Sample Calculations}

Although our model can be used for an arbitrary height of burst, our interest is in near-surface bursts, where shadowing by buildings and terrain is greatest. In this section we show sample calculations for near-surface bursts in two different urban venues: Oklahoma City, which has flat terrain, and San Francisco, which has significant terrain features.

\section{Oklahoma City}

For the Oklahoma City calculations ground zero is located at the approximate position of the truck bomb that destroyed the Murrah building, as shown in the aerial photo of Fig. 5. Figure 6 shows the calculated distribution of thermal radiation from a 10 -kt burst at 5 meters above the surface. Significant shadowing by the high rise buildings is apparent. The yellow color in the figures corresponds to an irradiation of 5 $\mathrm{cal} / \mathrm{cm}^{2}$, which is the approximate threshold for ignition of easily combustible materials such as loose paper. First degree skin burns occur at approximately $2 \mathrm{cal} / \mathrm{cm}^{2}$. Note that, except near the detonation, vertical surfaces receive much more heat than horizontal surfaces because of the angle of incidence of the radiation. Surfaces facing the detonation with no shadowing receive a total irradiation greater than $5 \mathrm{cal} / \mathrm{cm}^{2}$ out to a range of $2.1 \mathrm{~km}$, which includes all of the area shown in Fig. 6.

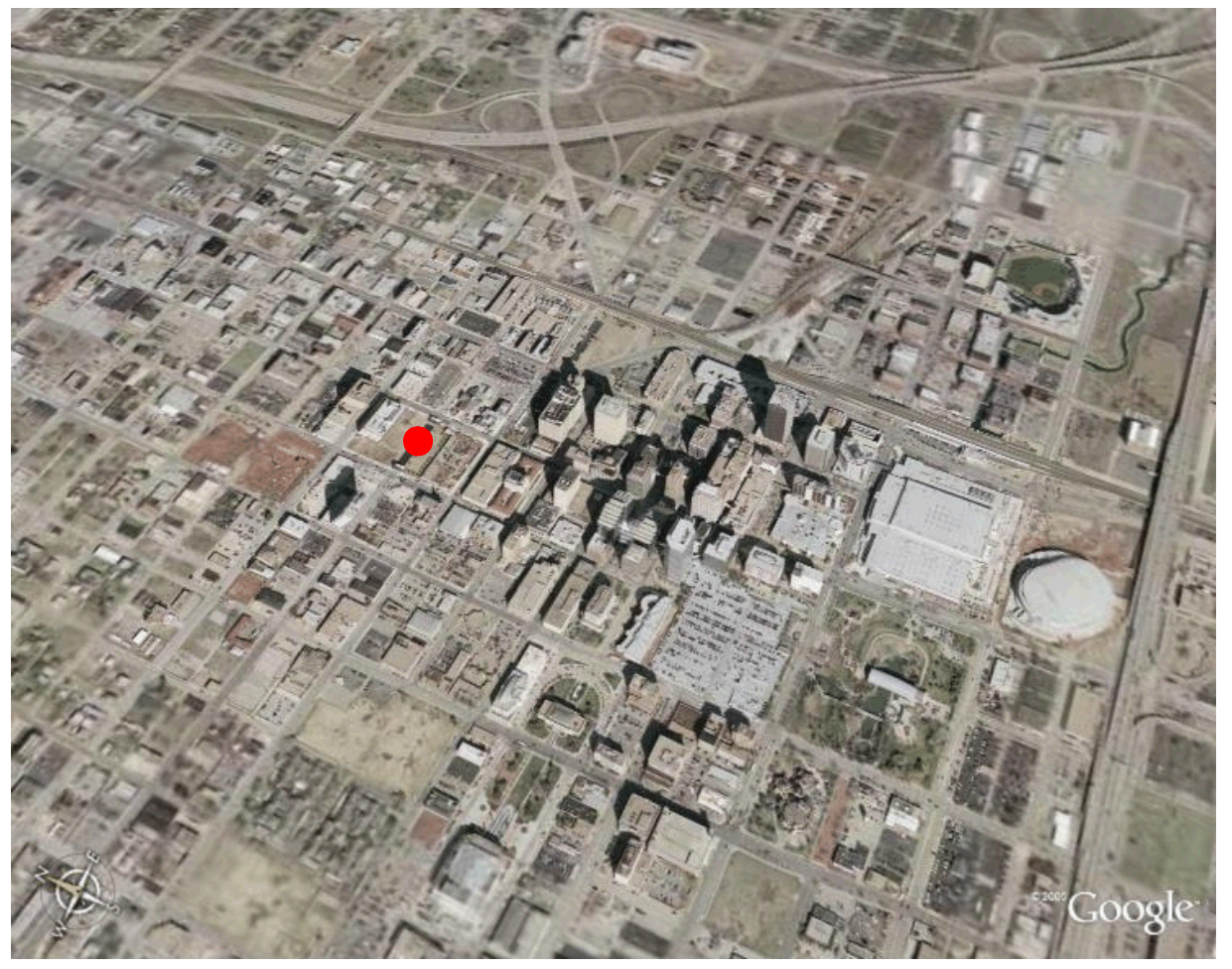

Fig. 5. Aerial photograph of Oklahoma City. The red circle indicates the location of ground zero for the thermal radiation calculations. 


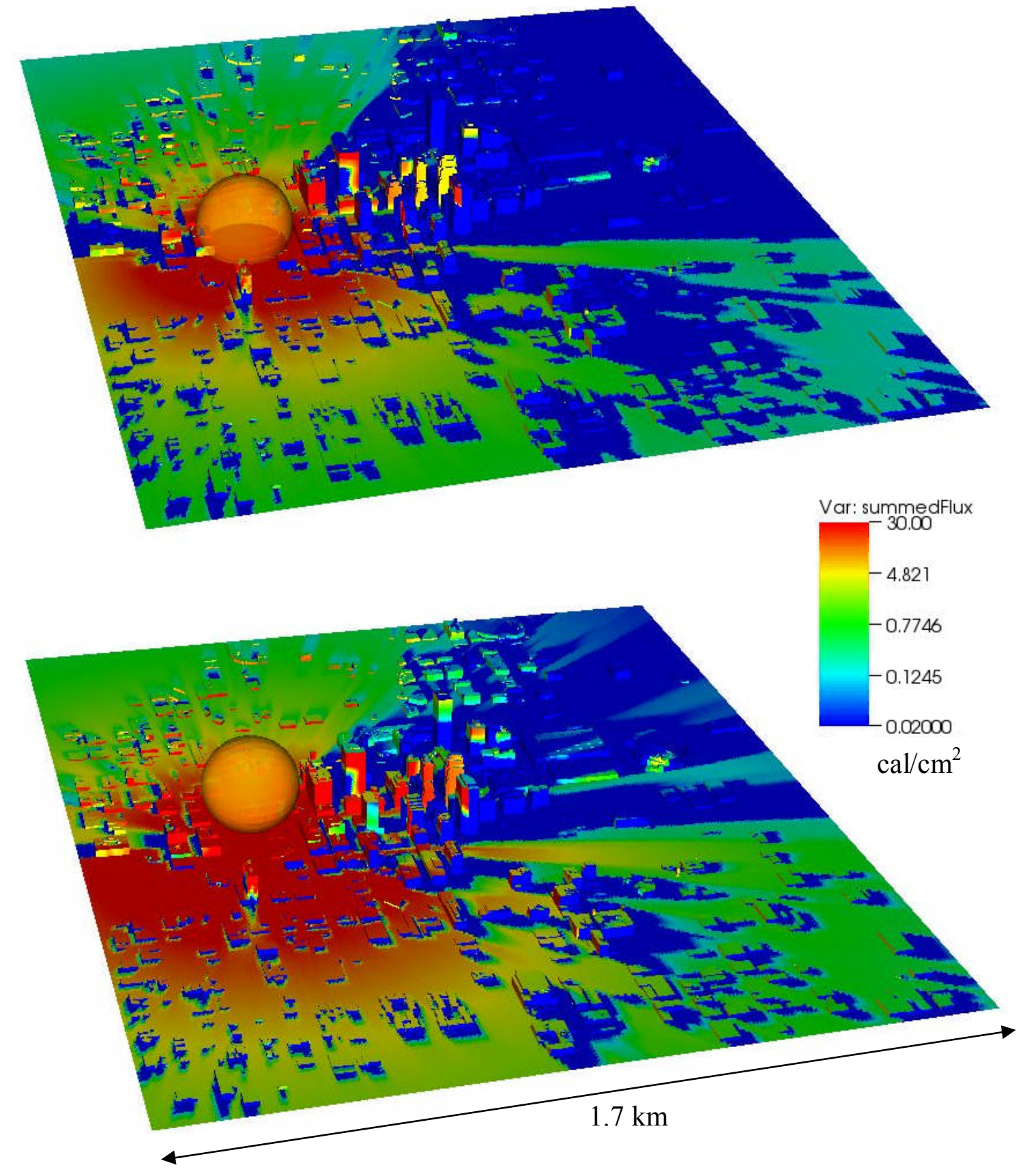

Fig. 6. Top: Heat deposition from a 10-kt surface detonation in Oklahoma City at the time of peak thermal power. The spherical bubble indicates the size and position of the radiating source at that time. Bottom: Heat deposition at 1 second after detonation over $90 \%$ of the total thermal energy has been radiated, and the fireball has risen roughly $100 \mathrm{~m}$. 
Figure 7 shows the heat distribution from a burst at a height of 300 meters with the same Oklahoma-City ground-zero location as the surface burst above. Note the reduced amount of shadowing. The ground is much hotter for the $300-\mathrm{m}$ burst than for the surface burst because of the different angle of incidence for the thermal radiation. The Oklahoma City calculations used 30 equally-spaced time steps and required approximately 1 hour of computer time with 16 parallel processors on the zeus machine at LLNL.

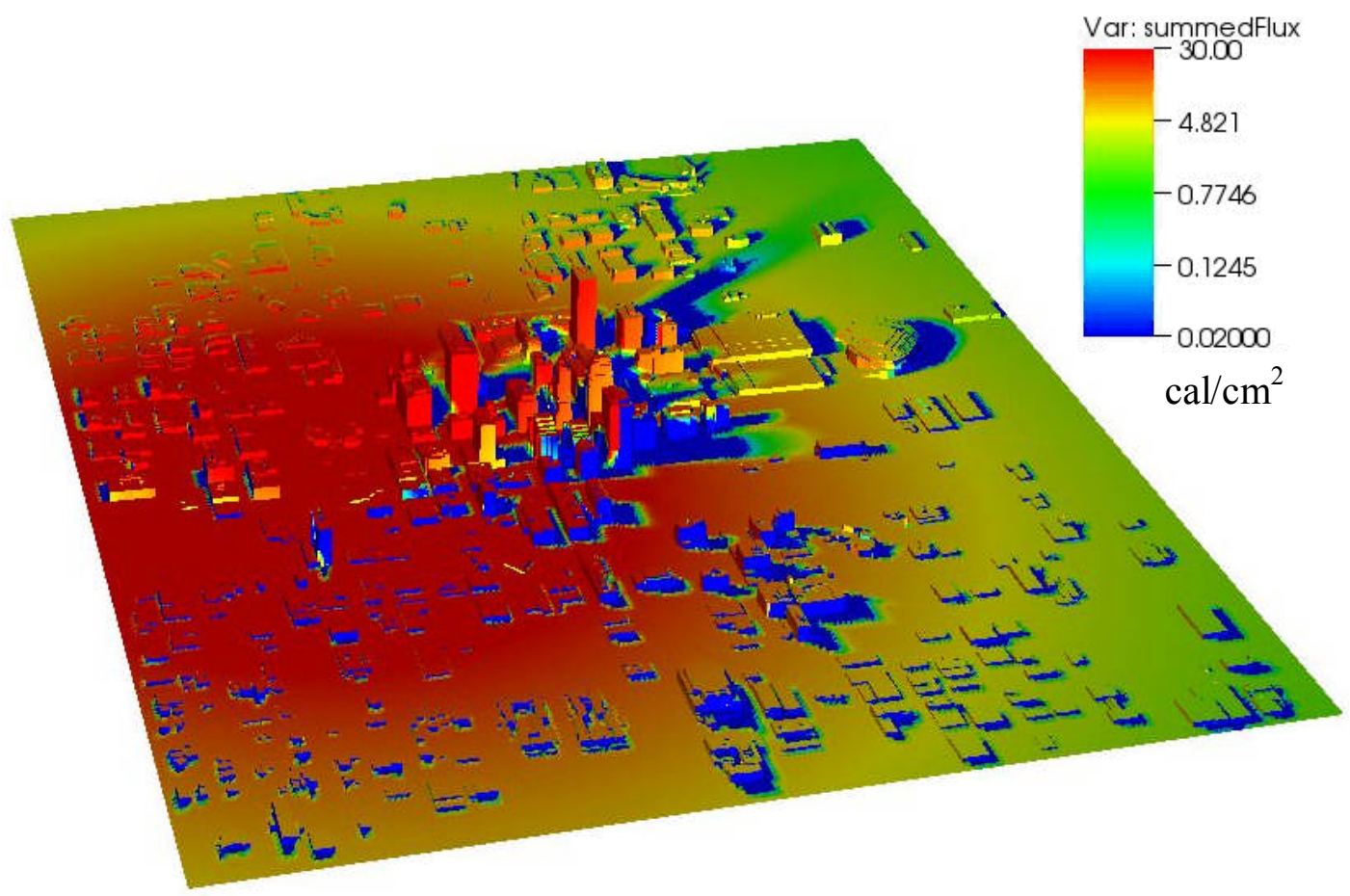

Fig. 7. Heat deposition in Oklahoma City at 1 second after a 10-kt detonation for a 300$\mathrm{m}$ height of burst. The color scale is the same as in Fig. 6 .

\section{San Francisco}

San Francisco has significant terrain features in addition to a cluster of tall buildings whose height is similar to the size of a 10-kt fireball. Figure 8 shows thermal radiation from a 10-kt detonation at both $5 \mathrm{~m}$ and $300 \mathrm{~m}$ above Market St. within the cluster of tall buildings. Remarkably, for a surface $(5 \mathrm{~m})$ detonation, most of the $5-\mathrm{km} \times$ $5-\mathrm{km}$ area shown is shielded from thermal radiation. For a $300-\mathrm{m}$ height of burst, a much large fraction of the urban area is heated. In the absence of shadowing or attenuation, the $2-\mathrm{cal} / \mathrm{cm}^{2}$ threshold for first degree burns occurs at a distance of $3.4 \mathrm{~km}$ for a $10-\mathrm{kt}$ detonation. This range includes most of the area shown in Fig.8. 

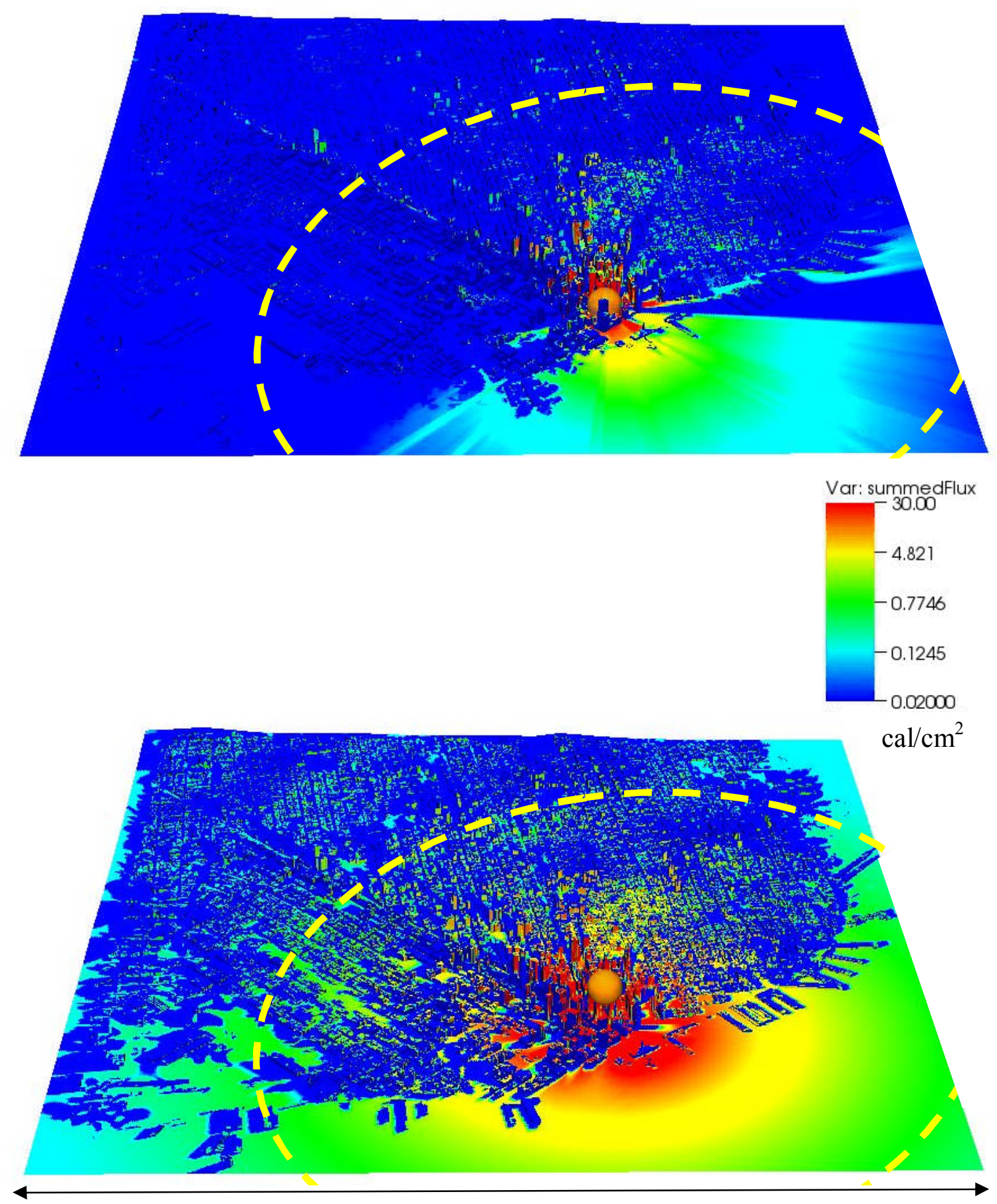

$5 \mathrm{~km}$

Fig. 8. Heat deposition in San Francisco at 1 second after a 10-kt detonation for a 5-m height of burst (top) and 300-m height of burst (bottom). At 1 second, over $90 \%$ of the thermal energy has been radiated. The fireball source is shown in its position at the time of peak radiated thermal power $(0.11 \mathrm{~s})$. The area shown is $5 \mathrm{~km} \times 5 \mathrm{~km}$. The yellow circle indicates the $5 \mathrm{cal} / \mathrm{cm}^{2}$ (ignition threshold) range for unobstructed irradiation. 


\section{Caveats and Conclusions}

There are several factors that could affect the accuracy of our thermal radiation model. Adjacent buildings and device emplacement could affect the early fireball and its thermal radiation. Although there is no accurate model for these effects, the additional mass and debris from an enclosed burst reduce the radiated thermal energy compared to a free air burst. Attenuation by fog, rain, and haze could be important at distances greater than the visibility range. In some cases, reflection from buildings and clouds could contribute significant heat to areas that are shielded from direct radiation. A general discussion of these effects can be found in Ref. [1].

An obvious next step (left for future work) would be a calculation of burn injuries and fires. Even without shadowing, the location of most of the urban population within buildings causes a substantial reduction in casualties compared to the unshielded estimates. Other investigators have estimated that the reduction in burn injuries may be greater than $90 \%$ due to shadowing and the indoor location of most of the population [6].

We have shown that common estimates of weapon effects that calculate a "radius" for thermal radiation are clearly misleading for surface bursts in urban environments. In many cases only a few unshadowed vertical surfaces, a small fraction of the area within a thermal damage radius, receive the expected heat flux.

In future work, our code could be extended to tally the total surface area receiving various amounts of heat, and to account for reflected radiation.

\section{References}

1. The Effects of Nuclear Weapons, edited by S. Glasstone and P. J. Dolan, U S Dept. of Defense (1977).

2. "RADFLO Physics and Algorithms," E. M. D. Symbalisty, J. Zinn, and R. W. Whitaker, LA-12988-MS (September, 1995).

3. "The Development and Testing of the Air Transport of Radiation Code Version 6 (ATR6).” D. C. Kaul et al., DNA-TR-91-237 (November, 1992).

4. Handbook of Nuclear Weapon Effects (EM-1), J. Northrop, DSWA (1996).

5. http://www.esri.com/

6. L. Davisson and M. Dombroski, private communication; "Radiological and Nuclear Response and Recovery Workshop: Nuclear Weapon Effects in an Urban Environment 2007,' M. Dombroski, B. Buddemeier, R. Wheeler, L. Davisson, T. Edmunds, L. Brandt, R. Allen, L. Klennert, and K. Law, UCRL-TR-XXXX (2007), in review. 